University of Wollongong

Research Online

Faculty of Engineering and Information

Faculty of Engineering and Information

Sciences - Papers: Part A

Sciences

$1-1-2016$

\title{
Radar imaging of stationary indoor targets using joint low-rank and sparsity constraints
}

Van Ha Tang

University of Wollongong, vht986@uowmail.edu.au

Abdesselam Bouzerdoum

University of Wollongong, bouzer@uow.edu.au

Son Lam Phung

University of Wollongong, phung@uow.edu.au

Fok Hing Chi Tivive

University of Wollongong, tivive@uow.edu.au

Follow this and additional works at: https://ro.uow.edu.au/eispapers

Part of the Engineering Commons, and the Science and Technology Studies Commons

Research Online is the open access institutional repository for the University of Wollongong. For further information contact the UOW Library: research-pubs@uow.edu.au 


\title{
Radar imaging of stationary indoor targets using joint low-rank and sparsity constraints
}

\begin{abstract}
This paper introduces a joint low-rank and sparsity-based model to address the problem of wall-clutter mitigation in compressed through-the-wall radar imaging. The proposed model is motivated by two observations that wall reflections reside in a low-rank subspace, and target signals tend to be sparse. In the proposed approach, the task of segregating target returns from wall reflections is formulated as a joint low-rank and sparsity constrained optimization problem. Here, the low rank constraint is imposed on the wall component and the sparsity constraint is used to model the target component. An iterative soft thresholding algorithm is developed to estimate a low-rank matrix of wall clutter and a sparse matrix of target reflections from a reduced measurement set. Once the wall and target components are estimated, the target signals are used for scene reconstruction. Experimental evaluation was conducted using real radar data. The results show that the proposed model is very effective at removing wall clutter and reconstructing the image of behind-the-wall targets from reduced measurements.
\end{abstract}

\section{Keywords}

joint, indoor, low, targets, rank, sparsity, constraints, stationary, imaging, radar

\section{Disciplines}

Engineering | Science and Technology Studies

\section{Publication Details}

V. Tang, A. Bouzerdoum, S. L. Phung \& F. Tivive, "Radar imaging of stationary indoor targets using joint low-rank and sparsity constraints," in 2016 IEEE International Conference on Acoustics, Speech and Signal Processing (ICASSP), 2016, pp. 1412-1416. 


\title{
RADAR IMAGING OF STATIONARY INDOOR TARGETS USING JOINT LOW-RANK AND SPARSITY CONSTRAINTS
}

\author{
V. H. Tang, A. Bouzerdoum, S. L. Phung, and F. H. C. Tivive \\ School of Electrical, Computer and Telecommunications Engineering, \\ University of Wollongong, NSW, 2522, Australia
}

\begin{abstract}
This paper introduces a joint low-rank and sparsity-based model to address the problem of wall-clutter mitigation in compressed through-the-wall radar imaging. The proposed model is motivated by two observations that wall reflections reside in a low-rank subspace, and target signals tend to be sparse. In the proposed approach, the task of segregating target returns from wall reflections is formulated as a joint low-rank and sparsity constrained optimization problem. Here, the low rank constraint is imposed on the wall component and the sparsity constraint is used to model the target component. An iterative soft thresholding algorithm is developed to estimate a low-rank matrix of wall clutter and a sparse matrix of target reflections from a reduced measurement set. Once the wall and target components are estimated, the target signals are used for scene reconstruction. Experimental evaluation was conducted using real radar data. The results show that the proposed model is very effective at removing wall clutter and reconstructing the image of behind-the-wall targets from reduced measurements.
\end{abstract}

Index Terms - Through-the-wall radar imaging, wall clutter mitigation, compressed sensing, low-rank matrix recovery, sparse reconstruction.

\section{INTRODUCTION}

Through-the-wall radar (TWR) imaging is intended for capturing scenes behind walls and other visually opaque materials. The ability to sense through enclosed structures is highly useful for numerous civilian and military applications, including search and rescue operations, surveillance and reconnaissance $[1,2]$. Imaging indoor scenes, however, is challenging due to strong front-wall electromagnetic (EM) returns. The front-wall EM reflections typically dominate those from targets, rendering target detection difficult or even impossible [3]. Furthermore, multiple reflections within the front wall produce reverberations which obscure the radar returns from weak targets. Hence, before scene reconstruction, the target signals need to be segregated from the wall returns to reduce clutter and reveal stationary indoor targets.

Conventionally, a scene image can be formed from a full measurement set using backprojection methods, such as delay-and-sum (DS) beamforming [4]. Recently, compressed sensing (CS) has been employed for fast data acquisition and accurate signal reconstruction from reduced data samples [5,6]. In TWR imaging, most CS techniques [7-11] assume that the wall returns can be completely removed before applying CS, or a background scene is available for suppressing the wall reflections. Very recently, wall mitigation techniques were investigated in the CS context [12-14]. In compressed TWR sensing, the same frequency observations may not be available at different antennas due to competing wireless services, intentional interferences, or radar jamming [15]. Therefore, most existing CSbased approaches for wall-clutter mitigation are performed in two stages. In the first stage, the antenna signals are recovered from reduced data samples using $\ell_{1}$ minimization [12], joint Bayesian sparse approximation [13], or block-sparse estimation [14]. In the second stage, the wall reflections are removed by applying clutter mitigation techniques, such as spatial filtering [16], or subspace projection $[17,18]$, prior to image formation.

In this paper, we propose a new approach for wall clutter mitigation and scene reconstruction in compressed TWR sensing. The proposed approach is inspired by two important observations that the wall radar returns lie in a low-rank subspace, and the target signals are sparse when represented in a proper basis. An optimization problem with low-rank and sparse constraints is proposed to separate the wall reflections from the target returns. Given a matrix of reduced measurements, the objective of the optimization problem is to jointly estimate a matrix of wall reflections and a matrix of target signals. In other words, it aims to decompose a matrix with missing entries into a low-rank matrix comprising wall returns and a sparse matrix containing target signals. Compared to the existing two-stage methods, the proposed approach is able to simultaneously segregate target signals from wall reflections, recover missing target returns, and produce clutter-free signals for image reconstruction in a unified framework.

The remainder of the paper is organized as follows. Section 2 introduces the TWR signal model. Section 3 describes the proposed joint low-rank and sparsity model for segregating wall returns and target signals. Section 4 presents experimental results and analysis. Section 5 gives concluding remarks.

\section{TWR SIGNAL MODEL}

Consider a monostatic stepped-frequency TWR system where a transceiver is placed at several scan positions parallel to the wall to synthesize a horizontal $M$-element linear antenna array. The scene is interrogated by transceiving a stepped-frequency signal comprising $N$ frequencies, equally spaced over the sensing bandwidth. Suppose that the scene contains $P$ targets placed behind the wall. Let $z_{n, m}$ denote the $n$-th frequency signal received at the $m$-th antenna location. The signal $z_{n, m}$ is modeled as the superposition of the wall reflection $z_{n, m}^{w}$ (including wall reverberations), target return $z_{n, m}^{t}$, and noise $v_{n, m}$ :

$$
z_{n, m}=z_{n, m}^{w}+z_{n, m}^{t}+v_{n, m} .
$$

The wall component $z_{n, m}^{w}$ is given by

$$
z_{n, m}^{w}=\sum_{l=1}^{L} \sigma_{w} a_{l} e^{-j 2 \pi f_{n} \tau_{m, w}^{l}},
$$


where $\sigma_{w}$ is the reflectivity of the wall, $L$ is the number of wall reverberations, $a_{l}$ is the path loss factor associated with the $l$-th wall return, and $\tau_{m, w}^{l}$ is the propagation delay of the $l$-th wall reverberation. The target return can be expressed as

$$
z_{n, m}^{t}=\sum_{p=1}^{P} \sigma_{p} e^{-j 2 \pi f_{n} \tau_{m, p}}
$$

where $\sigma_{p}$ is the reflectivity of the $p$-th target, and $\tau_{m, p}$ is the roundtrip travel time of the signal from the $m$-th antenna location to the $p$-th target.

For image formation, the scene is partitioned into a rectangular grid consisting of $Q$ pixels. Using DS beamforming, the complex amplitude of the $q$-th pixel can be computed as [4]

$$
I(q)=\frac{1}{M N} \sum_{m=1}^{M} \sum_{n=1}^{N} z_{n, m} \exp \left(j 2 \pi f_{n} \tau_{m, q}\right),
$$

where $\tau_{m, q}$ is the focusing delay between the $m$-th transceiver and the target located at the $q$-th pixel position. Applying DS beamforming or other backprojection methods to the radar signal given in Eq. (1) yields a scene image in which strong wall clutter obscures the targets of interest. To reveal the targets, wall reflections must be removed from the received signals before image formation; that is, ideally we want to replace $z_{n, m}$ in Eq. (4) by $z_{n, m}^{t}$ given in Eq. (3). If the full data volume is available, wall mitigation techniques $[3,16,17]$ can be applied directly to recover $z_{n, m}^{t}$. However, for practical compressed TWR sensing, we may have only a reduced measurement set acquired along the antenna array. Hence, the techniques that perform wall clutter mitigation within the CS context need to be investigated for indoor imaging. The next section describes the proposed joint low-rank and sparsity model for segregating target signals from wall returns.

\section{JOINT LOW-RANK AND SPARSITY MODEL}

This section presents the joint low-rank and sparse model for wall clutter mitigation and scene reconstruction in compressed TWR sensing. First, the received measurements along different antennas are arranged into a matrix. Then the task of separating wall reflections and target returns is cast as a low-rank and sparse constrained optimization model, where the nuclear-norm is used to enforce the low-rank property of the wall-clutter matrix and the $\ell_{1}$-norm is used to guarantee the sparsity of the target matrix. An iterative soft thresholding algorithm is developed to obtain the wall and target components. Finally, the target component is used for scene reconstruction.

\subsection{Problem formulation}

Let $\mathbf{Z}=\left[z_{n, m}\right], \mathbf{Z}^{w}=\left[z_{n, m}^{w}\right], \mathbf{Z}^{t}=\left[z_{n, m}^{t}\right]$, and $\boldsymbol{\Upsilon}=\left[v_{n, m}\right]$ denote the $N \times M$ matrices containing, respectively, the radar signals, the wall reflections, the target returns, and the noise received for all $N$ frequencies by all $M$ antennas. Equation (1) can be rewritten in matrix-form as

$$
\mathbf{Z}=\mathbf{Z}^{w}+\mathbf{Z}^{t}+\mathbf{\Upsilon}
$$

Now, the goal is to estimate $\mathbf{Z}^{w}$ and $\mathbf{Z}^{t}$ by decomposing the matrix $\mathbf{Z}$ into a low-rank matrix $\mathbf{Z}^{w}$ and a sparse matrix $\mathbf{Z}^{t}$, plus noise. This decomposition task is known as robust principal component analysis (RPCA) [19]. In RPCA model, given a data matrix, which is the superposition of a low-rank component and a sparse component, it is possible to recover both the low-rank and sparse components exactly by minimizing a weighted combination of the nuclear norm and $\ell_{1}$ norm. Let $\left\|\mathbf{Z}^{w}\right\|_{*}$ denote the nuclear-norm (i.e. the sum of the singular values of the matrix $\mathbf{Z}^{w}$ ) and let $\left\|\mathbf{Z}^{t}\right\|_{1}$ be the $\ell_{1}$-norm of $\mathbf{Z}^{t}$. Then, the low-rank component $\mathbf{Z}^{w}$ and sparse component $\mathbf{Z}^{t}$ can be estimated by solving the following optimization problem:

$$
\underset{\mathbf{Z}^{w}, \mathbf{Z}^{t}}{\operatorname{minimize}}\left\|\mathbf{Z}^{w}\right\|_{*}+\lambda\left\|\mathbf{Z}^{t}\right\|_{1} \quad \text { s. t. } \quad\left\|\mathbf{Z}-\left(\mathbf{Z}^{w}+\mathbf{Z}^{t}\right)\right\|_{2}<\epsilon,
$$

where $\|\cdot\|_{2}$ represents the Frobenius norm, $\lambda$ is a regularization parameter used to achieve a trade off between the low-rank and sparse constraints, and $\epsilon$ is a noise bound. The RPCA framework, however, is effective only if all the data measurements or entries of $\mathbf{Z}$ are available. In this case, the problem in Eq. (6) can be solved using convex optimization [20].

In compressed TWR sensing, instead of collecting all $(N \times M)$ data samples, only a reduce set containing $K$ measurements is obtained $(K \ll N \times M)$. Let $\boldsymbol{\Phi}$ be a selection matrix containing a single unit value in each row and each column. We denote by $\mathcal{A}$ : $\mathbb{C}^{M \times N} \rightarrow \mathbb{C}^{K}$ the linear operator mapping an $N \times M$ matrix $\mathbf{Z}$ into a $K \times 1$ vector $\mathbf{y}$,

$$
\mathbf{y}=\mathcal{A}(\mathbf{Z})=\mathbf{\Phi} \operatorname{vec}(\mathbf{Z}),
$$

where $\operatorname{vec}(\mathbf{Z})$ denotes the vectorization by stacking the matrix $\mathbf{Z}$ into a column vector. The compressed measurement vector $\mathbf{y} \in \mathbb{C}^{K}$ can be expressed as

$$
\mathbf{y}=\mathcal{A}(\mathbf{Z})=\mathcal{A}\left(\mathbf{Z}^{w}+\mathbf{Z}^{t}+\mathbf{\Upsilon}\right)
$$

Now we can recast the optimization problem in (6) as

$$
\underset{\mathbf{Z}^{w}, \mathbf{Z}^{t}}{\operatorname{minimize}}\left\|\mathbf{Z}^{w}\right\|_{*}+\lambda\left\|\mathbf{Z}^{t}\right\|_{1} \quad \text { s. t. } \quad\left\|\mathbf{y}-\mathcal{A}\left(\mathbf{Z}^{w}+\mathbf{Z}^{t}\right)\right\|_{2}<\epsilon .
$$

A greedy method, namely SpaRSC [21] has been proposed for solving the optimization problem (9). It combines ADMiRA [22], for low-rank matrix recovery, with CoSaMP [23], for sparse component recovery. This method, however, requires knowledge of the rank $R$ of matrix $\mathbf{Z}^{w}$ and the sparsity level $S$ of the sparse matrix $\mathbf{Z}^{t}$, which are typically unknown in practical TWR sensing problems. In [24], a template for first-order conic solvers (TFOCS) framework was adopted to estimate the low-rank and sparse components, but it is not robust in the presence of noise. To overcome these challenges, we introduce next an iterative algorithm to solve the optimization problem (9). The solution yields a low-rank matrix of wall returns and a sparse matrix of target signals.

\subsection{Optimization algorithm}

We propose an iterative algorithm to jointly recover a low-rank matrix and a sparse component given reduced data samples, see Eq. (9). Here, instead of assuming the sparsity in the signal domain, we incorporate a sparsifying dictionary $\mathbf{W}$ used to represent the signal matrix. Furthermore, the constrained optimization problem (9) is cast into a Lagrangian regularization form:

$$
\underset{\mathbf{Z}^{w}, \mathbf{Z}^{t}}{\operatorname{minimize}}\left\|\mathbf{y}-\mathcal{A}\left(\mathbf{Z}^{w}+\mathbf{Z}^{t}\right)\right\|_{2}+\lambda_{w}\left(\left\|\mathbf{Z}^{w}\right\|_{*}+\lambda\left\|\mathbf{W} \mathbf{Z}^{t}\right\|_{1}\right),
$$

or

$$
\underset{\mathbf{Z}^{w}, \mathbf{Z}^{t}}{\operatorname{minimize}}\left\|\mathbf{y}-\mathcal{A}\left(\mathbf{Z}^{w}+\mathbf{Z}^{t}\right)\right\|_{2}+\lambda_{w}\left\|\mathbf{Z}^{w}\right\|_{*}+\lambda_{t}\left\|\mathbf{W} \mathbf{Z}^{t}\right\|_{1},
$$


where $\lambda_{w}$ and $\lambda_{t}$ are, respectively, the regularization parameters for the low-rank and sparse components. To solve Problem (11), we introduce an iterative soft thresholding algorithm that efficiently shrinks the singular values of $\mathbf{Z}^{w}$ and the entries of the matrix $\mathbf{W} \mathbf{Z}^{t}$ towards zero. To this end, we define a thresholding operator

$$
\mathcal{T}_{\tau}(x)=\frac{x}{|x|}(|x|-\tau)_{+},
$$

where $x$ is a complex number, $\tau$ is a real number, and $(t)_{+}$is the positive part of $t$, i.e. $(t)_{+}=\max (t, 0)$. Extended to vectors and matrices, this shrinkage operator is applied entrywise. Using this shrinkage operator, a singular value thresholding (SVT) operator applied to $\mathbf{Z}^{w}$ is defined as

$$
\mathcal{S}_{\tau}\left(\mathbf{Z}^{w}\right)=\mathbf{U} \mathcal{T}_{\tau}(\boldsymbol{\Lambda}) \mathbf{V}^{H},
$$

where $\mathbf{Z}^{w}=\mathbf{U} \boldsymbol{\Lambda} \mathbf{V}^{H}$ is the singular value decomposition of $\mathbf{Z}^{w}$.

Given the compressed measurement vector $\mathbf{y}$, application of the iterative soft thresholding algorithm produces a series of estimates $\tilde{\mathbf{Z}}_{i}^{w}$ and $\tilde{\mathbf{Z}}_{i}^{t}$ that converge towards the true values $\mathbf{Z}^{w}$ and $\mathbf{Z}^{t}$. Here $\tilde{\mathbf{Z}}_{i}^{w}$ and $\tilde{\mathbf{Z}}_{i}^{t}$ denote, respectively, the estimates of matrices $\mathbf{Z}^{w}$ and $\mathbf{Z}^{t}$ at the $i$-th iteration. The soft thresholding algorithm can be summarized in the following steps:

1. Initialization: Set $\tilde{\mathbf{Z}}_{0}=\mathcal{A}^{T} \mathbf{y}$, where $\mathcal{A}^{T}$ is the adjoint operator of $\mathcal{A}, \tilde{\mathbf{Z}}_{0}^{w}=\tilde{\mathbf{Z}}_{0}, \tilde{\mathbf{Z}}_{0}^{t}=\mathbf{0}$, and $i=1$.

2. Singular-value soft thresholding:

$$
\tilde{\mathbf{Z}}_{i}^{w}=\mathcal{S}_{\lambda_{w}}\left(\tilde{\mathbf{Z}}_{i-1}-\tilde{\mathbf{Z}}_{i-1}^{t}\right)
$$

3. Soft thresholding in the transform domain:

$$
\tilde{\mathbf{Z}}_{i}^{t}=\mathbf{W}^{\dagger}\left(\mathcal{T}_{\lambda_{t}} \mathbf{W}\left(\tilde{\mathbf{Z}}_{i-1}-\tilde{\mathbf{Z}}_{i-1}^{w}\right)\right)
$$

where ${ }^{\dagger}$ denotes pseudo-inverse operator.

4. Data consistency and iteration:

If

$$
\frac{\left\|\tilde{\mathbf{Z}}_{i}^{w}+\tilde{\mathbf{Z}}_{i}^{t}-\left(\tilde{\mathbf{Z}}_{i-1}^{w}+\tilde{\mathbf{Z}}_{i-1}^{t}\right)\right\|_{2}}{\left\|\tilde{\mathbf{Z}}_{i-1}^{w}+\tilde{\mathbf{Z}}_{i-1}^{t}\right\|_{2}}<\delta
$$

terminate the algorithm,

Else,

$$
\begin{aligned}
\tilde{\mathbf{Z}}_{i} & =\tilde{\mathbf{Z}}_{i}^{w}+\tilde{\mathbf{Z}}_{i}^{t}-\mathcal{A}^{T}\left(\mathcal{A}\left(\tilde{\mathbf{Z}}_{i}^{w}+\tilde{\mathbf{Z}}_{i}^{t}\right)-\mathbf{y}\right) \\
i & \leftarrow i+1
\end{aligned}
$$

go to Step 2.

This iterative algorithm performs two main tasks: soft thresholding of singular values for low-rank estimation and iterative shrinkage for sparse reconstruction. It can be considered as a combination of SVT method for matrix completion [25] and an iterative shrinkage approach employed for sparse estimation [26]. In [25], it has been proven that the low-rank property is guaranteed, and the iterative SVT converges to an accurate low-rank estimate. It has also been shown in [26] that iterative shrinkage of signal coefficients in a transform domain promotes sparsity and converges for $\ell_{1}$-norm minimization problems. Since the proposed algorithm combines the SVT and shrinkage operators, it inherits its convergence properties from these two methods and leads to accurate estimates of low-rank matrix $\tilde{\mathbf{Z}}^{w}$ and sparse component $\tilde{\mathbf{Z}}^{t}$. Now, the estimated target component $\tilde{\mathbf{Z}}^{t}$ can be used for image formation. It is important to note here that the proposed model not only segregates the target signals from wall returns, but it also recovers the missing target signals in matrix $\tilde{\mathbf{Z}}^{t}$.

\section{EXPERIMENTAL RESULTS AND ANALYSIS}

In this section, we present the experimental results obtained using real radar data. Experimental analysis and comparison with existing compressed TWR imaging models are also provided.

\subsection{Experimental setup}

The proposed approach is evaluated on real radar data acquired with a radar system placed in front of a concrete wall of thickness $0.143 \mathrm{~m}$. A 57-element line array with an inter-element spacing of $0.022 \mathrm{~m}$ was placed at a standoff distance of $1.016 \mathrm{~m}$ away from the wall. A stepped-frequency signal between 0.7 and $3.1 \mathrm{GHz}$, with 3 $\mathrm{MHz}$ frequency step, was used to scan a scene containing a $0.39 \mathrm{~m}$ high and $0.28 \mathrm{~m}$ wide dihedral. The imaged scene, extending from $[0,4] \mathrm{m}$ in downrange and $[-2,2] \mathrm{m}$ in crossrange, is partitioned into an image of $100 \times 100$ pixels.

The proposed model requires a sparsifying dictionary $\mathbf{W}$ and regularization parameters $\lambda_{w}$ and $\lambda_{t}$. In all experiments, the dictionary $\mathbf{W}$ is constructed using modulated discrete prolate spheroidal sequences (DPSS) since the DPSS dictionary can represent bandpass radar signals more compactly than does the Fourier basis [14]. The algorithm is found to be robust for small regularization parameter values, which are set to $\lambda_{w}=\lambda_{t}=10^{-2}$. The algorithm terminates when the relative change of the solution is smaller than $\delta=10^{-8}$, see Step 4.

In the experiments, the image quality is evaluated using the target-to-clutter ratio (in $\mathrm{dB}$ ):

$$
\mathrm{TCR}=10 \log _{10}\left(\frac{\frac{1}{N_{t}} \sum_{q \in A_{t}}|I(q)|^{2}}{\frac{1}{N_{c}} \sum_{q \in A_{c}}|I(q)|^{2}}\right),
$$

where $A_{t}$ is the target region, $A_{c}$ is the clutter region defined as the entire image excluding the target region, $N_{t}$ and $N_{c}$ are the number of pixels in the target and clutter regions, respectively. The receiver operating characteristic (ROC) curve is used to compare the probability of target detection at different false alarm rates [2].

\subsection{Results and analysis}

In the first experiment, we evaluate the proposed model for the case when all measurements are available for image formation. Figure 1(a) shows the image of the dihedral formed using the full data without wall clutter mitigation. Clearly, the wall returns dominate the target signals, making target detection difficult. Figures 1(b) and 1(c) present the images formed after applying wall clutter mitigation using, respectively, spatial filtering [16] and subspace projection [3] techniques. It can be observed that the strong front wall clutter and its reverberations are suppressed, revealing the target. Figure 1(d) shows the image formed using the target signal obtained by the proposed joint low-rank and sparsity approach. In the formed image, clutter and background noise are further reduced, resulting in an improved TCR image.

In the second experiment, a reduced data set that accounts for only $15 \%$ of the full data volume was randomly selected for imaging the target. Since the wall-clutter mitigation techniques are not effective for compressed TWR imaging, we present here imaging results, shown in Figs. 2(a)-(b), obtained with a two-stage TWR imaging scheme [12] that first recovers radar signals independently along antennas using $\ell_{1}$ minimization and then applies wall clutter mitigation techniques. It can be observed that the target is detected, but the intensity of the target pixels is weak. For comparison, Figure 2(c) presents the image formed with the target component obtained using 


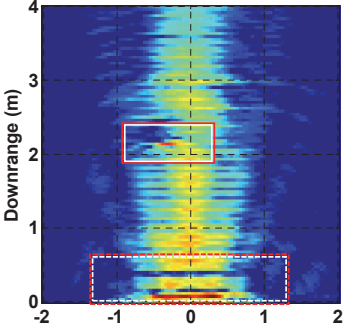

(a)

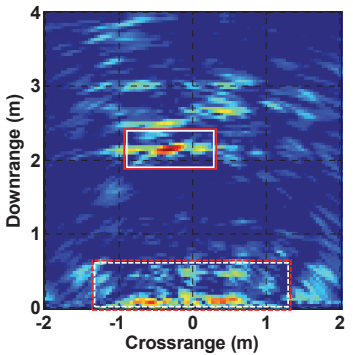

(c)

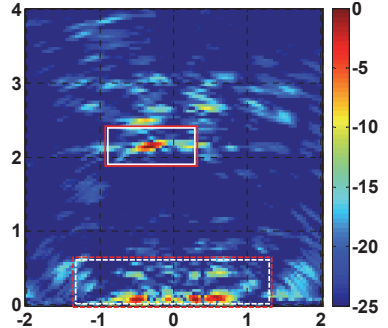

(b)

(d)

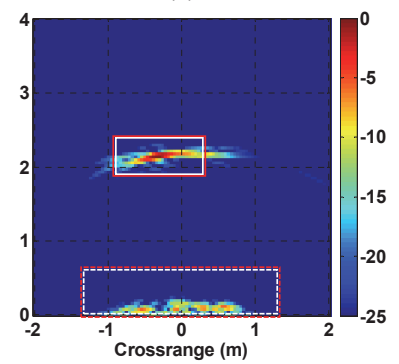

Fig. 1. Images formed with full measurements using: (a) raw data (TCR=9.42 dB), (b) spatial filtering (TCR=18.41 dB), (c) subspace projection $(\mathrm{TCR}=18.89 \mathrm{~dB})$, (d) proposed joint low-rank and sparsity model $(\mathrm{TCR}=24.03 \mathrm{~dB})$. Wall and target regions are indicated with dashed and solid rectangles, respectively.

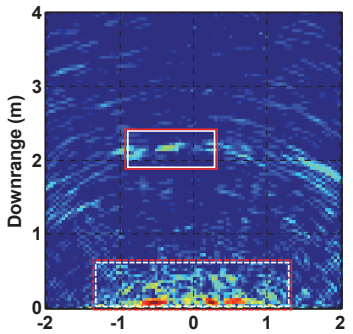

(a)

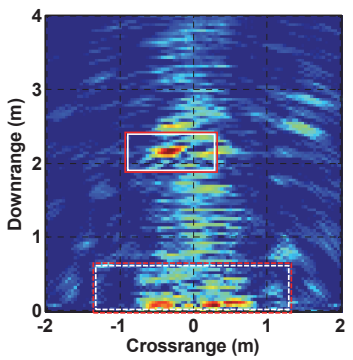

(c)

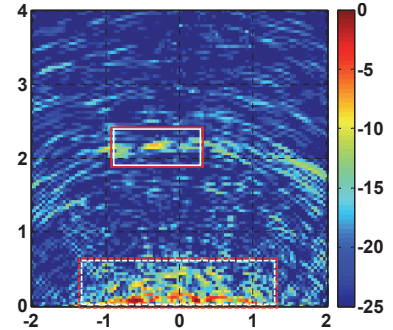

(b)

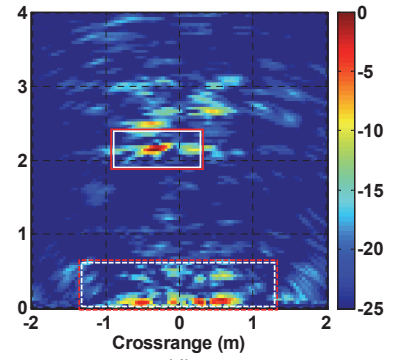

(d)
Fig. 2. Images formed with $15 \%$ frequency measurements using: (a) two-stage $\ell_{1}$ minimization \& spatial filtering (TCR=7.88 dB), (b) two-stage $\ell_{1}$ minimization $\&$ subspace projection $(\mathrm{TCR}=9.33$ dB), (c) target signals estimated with SpaRSC [21] (TCR=17.88 $\mathrm{dB})$, and (d) target signals reconstructed by the proposed algorithm $(\mathrm{TCR}=18.79 \mathrm{~dB})$.

SpaRSC method [21] to solve the low-rank and sparsity model. Note that this algorithm requires knowledge of the rank $R$ of the wall matrix and the sparsity level $S$ of the target signal matrix. Here these parameters were set to $R=2$ and $S=20$. We observe that the tar- get region is significantly enhanced. Figure 2(d) presents the image formed using the target component reconstructed by the proposed iterative algorithm. It is clear that the image quality is enhanced and the clutter is further attenuated.

Figure 3 illustrates the ROC curves of the images formed after applying different wall clutter mitigation approaches. We observe that the joint low-rank and sparsity model significantly enhances target detection, especially when the number of data samples is significantly reduced. The improvement in image quality and target detection can be justified by the fact that the proposed model uses both the low-rank and sparse representations to capture the underlying structures of the TWR signals. As a result, the proposed model is able to jointly separate wall and target components and reconstruct the target signals, which are used for image formation.

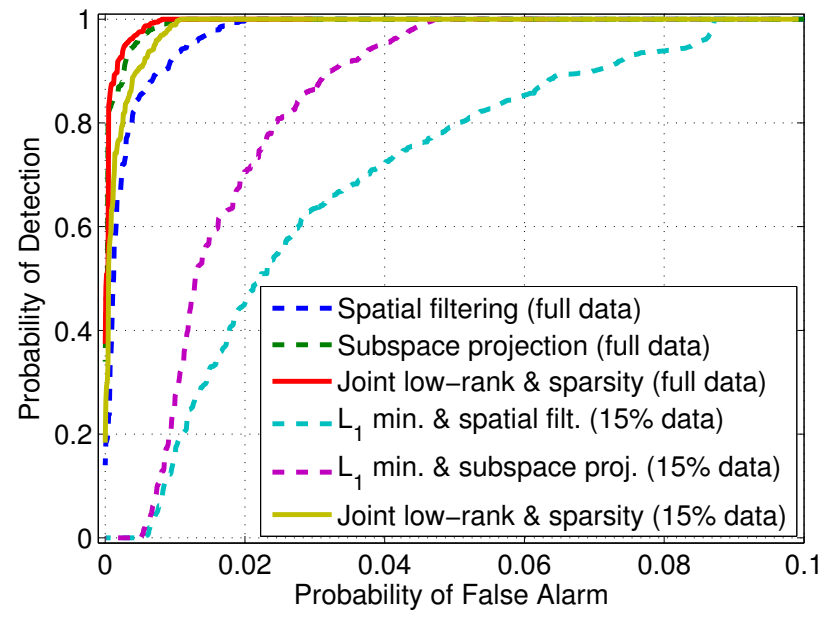

Fig. 3. ROC curves for target detection from images formed by applying different wall-clutter suppression methods. See electronic color figure.

\section{CONCLUSION}

This paper presented a joint low-rank and sparsity-based approach for wall clutter mitigation and target image formation. A constrained optimization model is formulated where the nuclear-norm is used to enforce the low-rank condition on the matrix of wall returns and $\ell_{1}$-norm is employed to control the sparsity of target signals. An iterative soft thresholding algorithm is proposed to estimate the wallclutter and target components from compressed radar measurements. Experimental results show that the proposed approach enhances the target-to-clutter ratio and improves target detection even when the number of measurements is significantly reduced.

\section{Acknowledgments}

The authors would like to thank Dr. Moeness G. Amin and Dr. Fauzia Ahmad from the Center of Advanced Communications at Villanova University, Villanova, PA, USA, for providing the experimental data. This work is supported by a grant from the Australian Research Council (ARC). 


\section{REFERENCES}

[1] M. G. Amin (Ed.), Through-The-Wall Radar Imaging. Boca Raton, FL: CRC Press, 2010.

[2] C. H. Seng, A. Bouzerdoum, M. G. Amin, and S. L. Phung, "Probabilistic fuzzy image fusion approach for radar through wall sensing," IEEE Trans. Image Processing, vol. 22, no. 12, pp. 4938-4951, Dec. 2013.

[3] F. H. C. Tivive, A. Bouzerdoum, and M. G. Amin, "An SVDbased approach for mitigating wall reflections in through-thewall radar imaging," Proc. IEEE Radar Conf., pp. 519-524, Kansas City, MO, 23-27 May 2011.

[4] F. Ahmad, M. G. Amin, and S. A. Kassam, "Synthetic aperture beamformer for imaging through a dielectric wall," IEEE Trans. Aerospace and Electronic Systems, vol. 41, no. 1, pp. 271-283, Jan. 2005.

[5] D. L. Donoho, "Compressed sensing," IEEE Trans. Information Theory, vol. 52, no. 4, pp. 1289-1306, Apr. 2006.

[6] E. J. Candes, J. Romberg, and T. Tao, "Stable signal recovery from incomplete and inaccurate measurements," Communications on Pure and Applied Mathematics, vol. 59, no. 8, pp. 1207-1223, Aug. 2006.

[7] Q. Huang, L. Qu, B. Wu, and G. Fang, "UWB through-wall imaging based on compressive sensing," IEEE Trans. Geoscience and Remote Sensing, vol. 48, no. 3, pp. 1408-1415, Mar. 2010.

[8] Y.-S. Yoon and M. G. Amin, "Through-the-wall radar imaging using compressive sensing along temporal frequency domain," Proc. IEEE Int. Conf. Acoustics, Speech, and Signal Processing, pp. 2806-2809, Dallas, TX, 14-19 Mar. 2010.

[9] M. Leigsnering, C. Debes, and A. M. Zoubir, "Compressive sensing in through-the-wall radar imaging," Proc. IEEE Int. Conf. Acoustics, Speech, and Signal Processing, pp. 40084011, Prague, Czech Republic, 22-27 May 2011.

[10] J. Yang, A. Bouzerdoum, F. H. C. Tivive, and M. G. Amin, "Multiple-measurement vector model and its application to through-the-wall radar imaging," Proc. IEEE Int. Conf. Acoustics, Speech and Signal Processing, pp. 2672-2675, Prague, Czech Republic, 22-27 May 2011.

[11] V. H. Tang, A. Bouzerdoum, and S. L. Phung, "Two-stage through-the-wall radar image formation using compressive sensing," Journal of Electronic Imaging, vol. 22, no. 2, pp. 021 006.1-021 006.10, Apr.-Jun. 2013.

[12] E. Lagunas, M. G. Amin, F. Ahmad, and M. Najar, "Joint wall mitigation and compressive sensing for indoor image reconstruction," IEEE Trans. Geoscience and Remote Sensing, vol. 51, no. 2, pp. 891 - 906, Feb. 2013.

[13] V. H. Tang, A. Bouzerdoum, S. L. Phung, and F. H. C. Tivive, "Enhanced wall clutter mitigation for through-the-wall radar imaging using joint Bayesian sparse signal recovery," Proc. IEEE Int. Conf. Acoustics, Speech and Signal Processing, pp. 7804-7808, Florence, Italy, 4-9 May 2014.
[14] F. Ahmad, J. Qian, and M. G. Amin, "Wall clutter mitigation using discrete prolate spheroidal sequences for sparse reconstruction of indoor stationary scenes," IEEE Trans. Geoscience and Remote Sensing, vol. 53, no. 3, pp. 1549-1557, March 2015.

[15] F. Ahmad and M. G. Amin, "Partially sparse reconstruction of behind-the-wall scenes," Proc. SPIE Compressive Sensing, pp. 83 650W.1-83 650W.9, Maryland, USA, 23-27 Apr. 2012.

[16] Y.-S. Yoon and M. G. Amin, "Spatial filtering for wall-clutter mitigation in through-the-wall radar imaging," IEEE Trans. Geoscience and Remote Sensing, vol. 47, no. 9, pp. 31923208, Sept. 2009.

[17] F. H. C. Tivive and A. Bouzerdoum, "An improved SVDbased wall clutter mitigation method for through-the-wall radar imaging," Proc. IEEE Workshop on Signal Processing Advances in Wireless Communications, pp. 430-434, Darmstadt, Germany, 16-19 June 2013.

[18] F. H. C. Tivive, A. Bouzerdoum, and M. G. Amin, "A subspace projection approach for wall clutter mitigation in through-thewall radar imaging," IEEE Trans. Geoscience and Remote Sensing, vol. 53, no. 4, pp. 2108-2122, Apr. 2015.

[19] E. J. Candes, X. Li, Y. Ma, and J. Wright, "Robust principal component analysis?" Journal of the ACM, vol. 58, no. 3, pp. 1-37, May 2011.

[20] V. Chandrasekaran, S. Sanghavi, P. A. Parrilo, and A. S. Willsky, "Rank-sparsity incoherence for matrix decomposition," SIAM Journal on Optimization, vol. 21, no. 2, pp. 572-596, June 2011.

[21] E. W. Andrew, C. S. Aswin, and R. Baraniuk, "SpaRCS: Recovering low-rank and sparse matrices from compressive measurements," Proc. Advances in Neural Information Processing Systems, pp. 1089-1097, Granada, Spain, 12-17 Dec. 2011.

[22] K. Lee and Y. Bresler, "ADMiRA: Atomic decomposition for minimum rank approximation," IEEE Trans. Information Theory, vol. 56, no. 9, pp. 4402-4416, Sep. 2010.

[23] D. Needell and J. A. Tropp, "CoSaMP: Iterative signal recovery from incomplete and inaccurate samples," Applied and Computational Harmonic Analysis, vol. 26, no. 3, pp. 301321, May 2009.

[24] J. Wright, A. Ganesh, K. Min, and Y. Ma, "Compressive principal component pursuit," Information and Inference, vol. 2, pp. 32-68, Mar. 2013.

[25] J.-F. Cai, E. J. Candes, and Z. Shen, "A singular value thresholding algorithm for matrix completion," SIAM Journal on Optimization, vol. 20, no. 4, pp. 1956-1982, Mar. 2010.

[26] I. Daubechies, M. Defrise, and C. D. Mol, "An iterative thresholding algorithm for linear inverse problems with a sparsity constraint," Communications on Pure and Applied Mathematics, vol. 57, no. 11, pp. 1413-1457, Nov. 2004. 